\title{
EAI Endorsed Transactions

\section{Design of a Novel Intelligent Framework for Finding Experts and Learning Peers in Open Knowledge Communities}

\author{
Pengfei $\mathrm{Wu}^{1,2,3}$, Shengquan $\mathrm{Yu}^{1,2, *}$ \\ ${ }^{1}$ School of Educational Technology, Faculty of Education, Beijing Normal University, China \\ ${ }^{2}$ The Joint Laboratory for Mobile Learning, Ministry of Education-China Mobile Communications Corporation, China \\ ${ }^{3}$ Library, Shijiazhuang University, China
}

\begin{abstract}
Open knowledge communities (OKCs) are computer supported collaborative learning environments that provide opportunities for social knowledge construction, collaboration, participation and communication for ubiquitous learning and informal learning. However, with the rapid expanding of learning content resources and users, it is difficult for learners to find the right persons they need as knowledge experts and learning peers in OKCs using traditional search engines. To solve this problem, the paper presents a novel intelligent framework for finding the right experts and learning peers based on social knowledge network. After describing the architectural details of the framework, the authors elaborate on the intelligent framework design, the work principle, and mechanism of each module. Finally, the authors conclude by showing the learning application scenarios of this intelligent framework.
\end{abstract}

Keywords: OKCs, right persons finding, SKN, ontology, linked data, SWRL, LDA, SNA, Learning Cell Knowledge Community.

Received on 30 May 2015, accepted on 10 June 2015, published on 16 June 2015

Copyright (C) 2015 S. Yu and P. Wu, licensed to ICST. This is an open access article distributed under the terms of the Creative Commons Attribution licence (http://creativecommons.org/licenses/by/3.0/), which permits unlimited use, distribution and reproduction in any medium so long as the original work is properly cited.

doi: 10.4108/fiee.1.2.e4

\section{Introduction}

Computer supported collaborative learning environments offer good functions for social knowledge collaboration, participation, and communication among learners and teachers. Open knowledge communities (OKCs) are online and open computer supported collaborative learning environments, which provide excellent opportunities for users to take part in collaborative knowledge authoring and sharing and exchanging knowledge, experience, and resources [1]. Better person-to-person interaction, discussion, and collaboration in OKCs will not only improve learning performance [2, 3, 4, 5], but also effectively eliminate learners' feelings of loneliness so as to improve learning satisfaction and motivation [6, 7]. However, with

${ }^{*}$ Corresponding author. Email: yusq@bnu.edu.cn the rapid expansion of learning content resources and users in OKCs, it is difficult for learners to find the right persons they need as knowledge experts and learning peers using traditional search engines.

We believe that providing the right expert- or peerfinding service for learners is vital for their smart learning, because the right experts or learning peers may offer authoritative, trustworthy knowledge and collective intelligence for learning. Based on this assumption, the paper presents an intelligent framework that uses hybrid technologies to find the right people in OKCs. We first describe the architectural details of this framework, then elaborate on the design of this intelligent framework, the work principles, and the mechanism of each module.

This paper is organized as follows: Section 2 presents related works as the theoretical background. Section 3 
presents our statement of the problem that needs research. Section 4 describes the definition of the social knowledge network. Section 5 describes this intelligent people-finding framework. The learning application scenarios of the intelligent framework are demonstrated in Section 6 . Finally, Section 7 presents our conclusions and plans for future work.

\section{Related Works}

\subsection{Experts Finding}

Experts finding is also called expert search or expert location that is aimed at discovering the relevant experts with authority knowledge in certain subject areas. TREC (Text Retrieval Conference) set up experts finding task in 2005 [8]. There are many studies on experts finding, which is to find persons with higher expertise, experiences, or skills in organizations and open online communities.

The earliest experts finding systems for organizations focused on expertise identification based on the experts' skills stored in a structure database [9, 10]. With the rapid expansion of information in the Internet, experts finding for open online communities have attracted more studies [11].

Many approaches and algorithms are proposed to find experts. The language model is used to calculate probability between query topics and candidate experts [12, 13, 14]. The topic-sensitive probabilistic model is proposed to find experts in questioning-answering communities [15]. Through the construction of the users' social network, methods based on social link network analysis have achieved expert-finding in open communities (such as Yahoo! Answers or Java forums) and enterprise organization [16]. Ontology can provide formal and sharable representation of knowledge. Ontologies-based experts finding method is also proposed to improve the search results [17, 18, 19]. At the same time, several hybrid methods have been proposed to find experts using language model, topic model, and social network linked analysis [20, 21, 22].

Previous studies provide approaches for the right people finding in OKCs. However, these studies mainly focus on finding people from the view of knowledge while ignore the different learning needs and roles of different users in OKCs.

\subsection{Social and Semantic Web in Education}

Web 2.0 technologies or social web such as blog, wiki, QQ (an instant communication tool that is popular in China) offering various social interaction techniques [23] have been changing the web from a single knowledge network to the integration of social networks and knowledge networks, which are generated and evolved by social, collective and collaborative interaction and participation among learners and teachers. It enables people not only to create, collaborate, interact, share, annotate, and exchange knowledge [24], but also to communicate and to share with others through knowledge or people. Using Web 2.0 technologies, learners with different learning interests and prior knowledge can flexibly acquire knowledge, skills, experience and collaboratively construct their own knowledge network through social learning interactions [25]. However, as for social web, ambiguity of meanings and human-understandable meanings not machineunderstandable meanings are the two big problems [26].

Semantic web technologies such as ontologies and RDF (Resource Description Framework) can provide an explicit approach to the formal and sharable representation of knowledge that aim to facilitate web-scale data interlinking and interoperability and make the web more machineunderstandable [27], because they guarantee high level of expressiveness, flexibility, and extensibility of the represented knowledge [28]. However, the semantic web cannot work alone and it needs society-scale applications [29]. Semantic web technologies offer a feasible approach to resolving social web problem through structured knowledge and machine-understandable representation. When the social web works together with semantic web integrating social interactions and explicit, semantically rich knowledge representations to form a web of collective knowledge systems in e-learning [30], it can offer excellent learning services for teachers and students.

\section{Research Question}

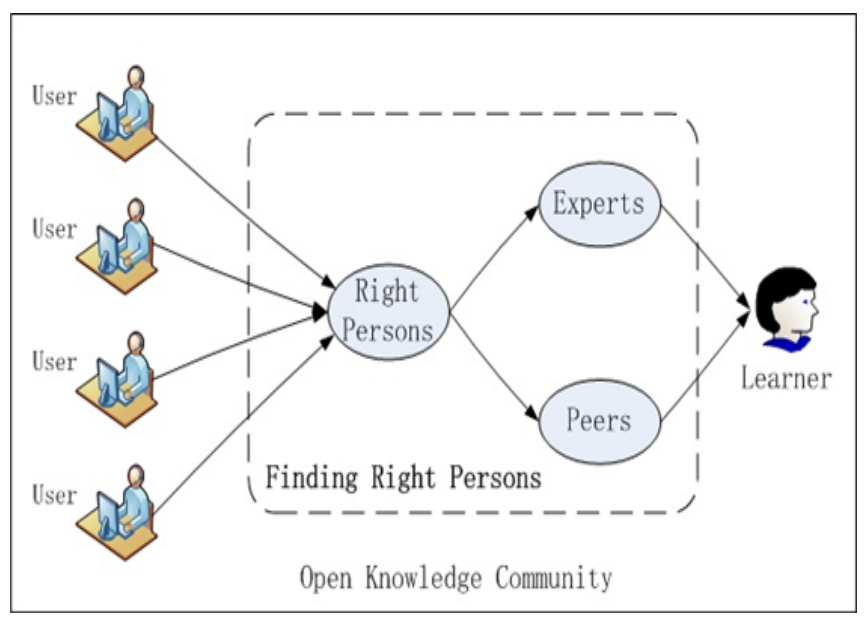

Figure 1. Research statement of finding right persons

Our primary research question is how to find the right experts and peers for each learner efficiently and effectively in OKCs. In the OKCs environment (as shown in Figure 1), users and knowledge points are linked through various social learning interactions. In the process of social learning interactions, a user in OKCs has different roles such as an expert, a peer, a learner, and so on. For example, when a user creates a lot of knowledge relevant to Java 
Programming, likely the user is an expert in Java Programming. As learners, they have different learning needs in different context. Sometimes, learners hope to find the authoritative knowledge experts relevant to specific topics to solve problems in learning process. Sometimes, learners hope to find peers who have the same interests for discussion and communication. Therefore, as for learners, experts and peers are the right people to interact with for better learning. Because of this, our study aimed to address how to find the right experts and peers for each learner efficiently and effectively in OKCs. This research question is depicted visually in Figure 1.

\section{Social Knowledge Network}

A general network consists of nodes and connections, with nodes representing entities and connections representing relationships between entities. A social network $(\mathrm{SN})$ is viewed as a special network that consists of a finite set of actors $(\mathrm{P})$ and relations between them (P2P) [31]. SN focuses on relations between humans. A knowledge network $(\mathrm{KN})$ is also a special network that is composed of knowledge (K) and connections between knowledge (K2K). $\mathrm{KN}$ focuses on knowledge and relations between knowledge nodes.

In open knowledge communities (OKCs), people share knowledge with each other through various learning interactions in different learning contexts. Social knowledge network (SKN) may be viewed as the integration of SN and $\mathrm{KN}$, which consists of people and knowledge as nodes and connections between them (PKR). These connections are composed of relationships among people (P2P), relationships between people and knowledge, (P2K) and relationships among knowledge (K2K).
Table 1. A Comparison among SN, KN and SKN

\begin{tabular}{llll}
\hline Characteristics & SN & KN & SKN \\
\hline Nodes & P & K & P \& K \\
Connections & P2P & K2K & PKR \\
\hline
\end{tabular}

For nice explanation of terms, we define the social knowledge network as follows:

Definition (Social Knowledge Network). SKN $=<\mathrm{N}$, R, L > where

- $\mathrm{N}=<\mathrm{PN}, \mathrm{KN}>$ represents the collection of nodes, where PN represents a collection of person nodes, KN represents a collection of knowledge nodes;

- $\mathrm{R}=<\mathrm{P} 2 \mathrm{P}, \mathrm{P} 2 \mathrm{~K}, \mathrm{~K} 2 \mathrm{~K}>$ represents the collection of relations, where the $\mathrm{P} 2 \mathrm{P}$ is a collection of relations among persons. $\mathrm{P} 2 \mathrm{~K}$ represents a collection of relation between persons and knowledge, $\mathrm{K} 2 \mathrm{~K}$ represents a collection of the relationship between knowledge and knowledge;

- $\mathrm{L}: \mathrm{R} \rightarrow \mathrm{N}$ represents correlation function, the associated relationships with person and knowledge.

\section{RPFinder: An Intelligent Finding Framework}

The intelligent right people-finding framework is called RPFinder and it includes seven logical components: SKN Construction, RPs Generator, Query Semantic Parser, Semantic Matching, Semantic Reasoner, RPs Ranker, and RPs Finding GUI. The architecture of RPFinder is illustrated in Figure 2.

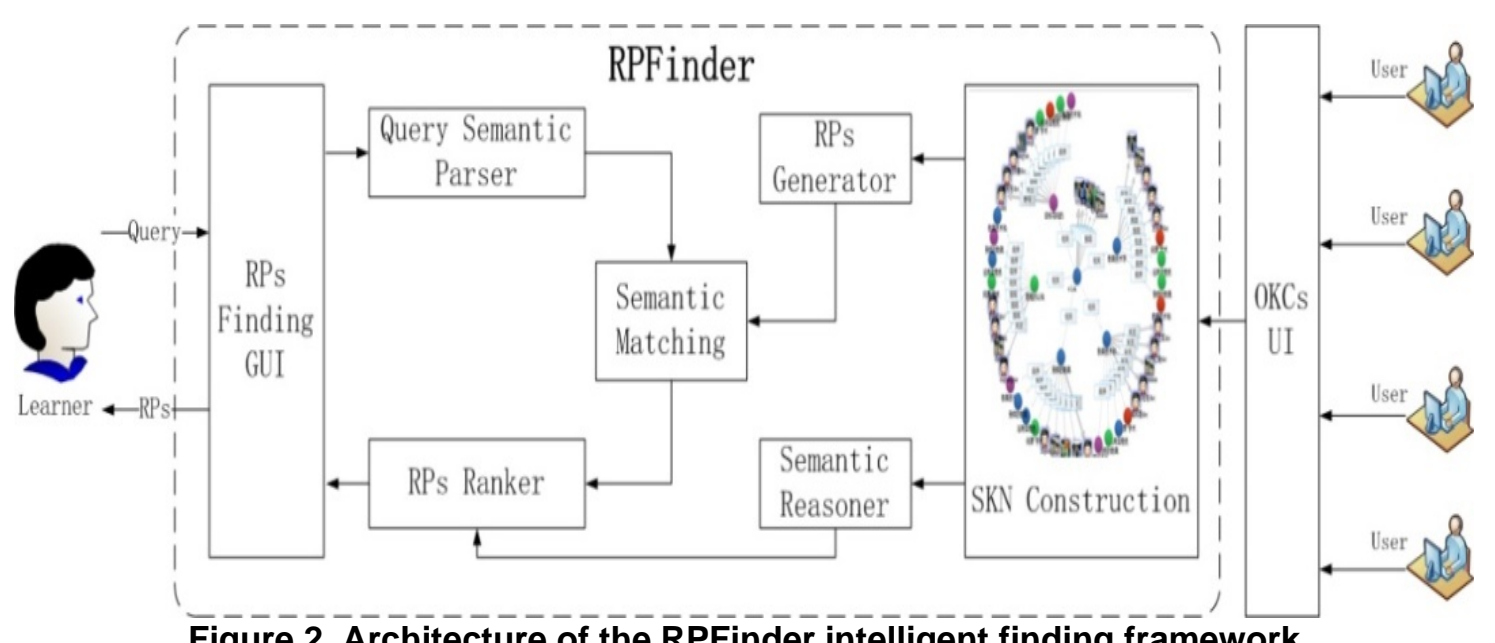

Figure 2 . Architecture of the RPFinder intelligent finding framework

\subsection{SKN Construction}

The aim of SKN Construction is to build a linked data network by semantics. In OKCs, people and knowledge are connected with each other through various social learning 
interactions in different learning contexts. Due to various learning interactions between people and knowledge, OKC forms a social knowledge network (SKN). SKN is a composite representation of graph model of a social network and knowledge network, specifically with RDF semantic graphical representation.

Ontology is a semantic organization technology for explicit and formal description about concepts, properties, and relationships providing high level meaning. Ontologies are important for the SKN Construction. We use two ways to build the ontologies. One way is to construct an ontology called SKNO (Social Knowledge Network Ontology) for explicitly and formally describing people, knowledge and their social interactive relations in OKCs from the learning perspective. A part of class hierarchy of the SKNO is illustrated in Figure 3. SKNO consists of three type classes: Knowledge, Person and PKR. Knowledge is an abstract concept including different learning resources such as a learning object, a learning tool, a learning cell and so on. Person is a concept to represent actor involved in learning process that consists of three sub-concepts: Learner, Peer and Expert. PKR (Person Knowledge Relationship) is composed of three type classes $P 2 P$ (Person to Person Relationship), $P 2 K$ (Person to Knowledge Relationship) and $K 2 K$ (Knowledge to Knowledge Relationship) representing different relationships between Person and Knowledge.

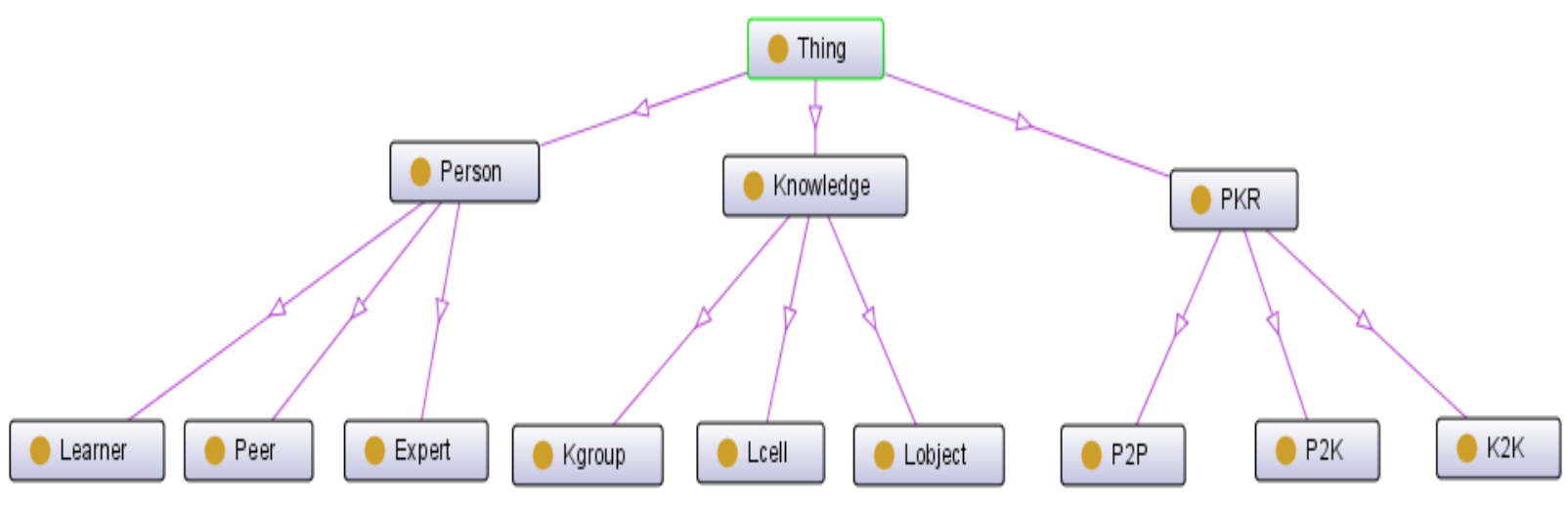

Figure 3. A Protégé screenshot representing a part of class hierarchy of the SKNO

Figure 4 is an extract of the SKNO properties. Properties $p 2 k p, p 2 p p$ and $k 2 k p$ are sub-properties of the root property pkrp.

Another way is to utilize and extend the existed ontologies such as LOM (Learning Object Metadata) for describing the learning content, DC (Dublin Core Metadata) for general web resources description, SKOS (Simple Knowledge Organization System) for relevant concepts and taxonomy description, FOAF (Friend-of-a-Friend) for people description, and so on.

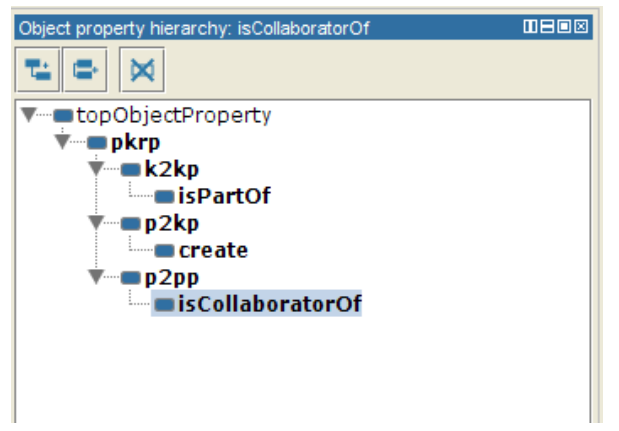

\section{Figure 4. A Protégé screenshot representing a part of properties hierarchy of the SKNO}

Linked data facilitates web-scale data interlinking and realization of semantic knowledge network based on a set of well-established principles and W3C standard technologies, e.g. RDF, SPARQL, and HTTP URIs [32].

SKN Construction is aimed at generating social knowledge network employing the above ontologies and linked data technologies to link person and knowledge from the OKCs log. SKN is a RDF graph for storing semantic and linked learning data.

Figure 5 depicts an example of SKN. In Figure 5, for example, the learner Tom is an instance of Person in SKNO and connected by the property create to the concept Information Retrieval Model instance of the class Knowledge, meaning that student Tom has created a learning resource labelled Information Retrieval Model. The property isPartOf between Boolean Model and Information Retrieval Model describes relationship about two instances of the class Knowledge. The property isCollaboratorOf between Jack and Tom describes relationship about two instances of the class Person. 


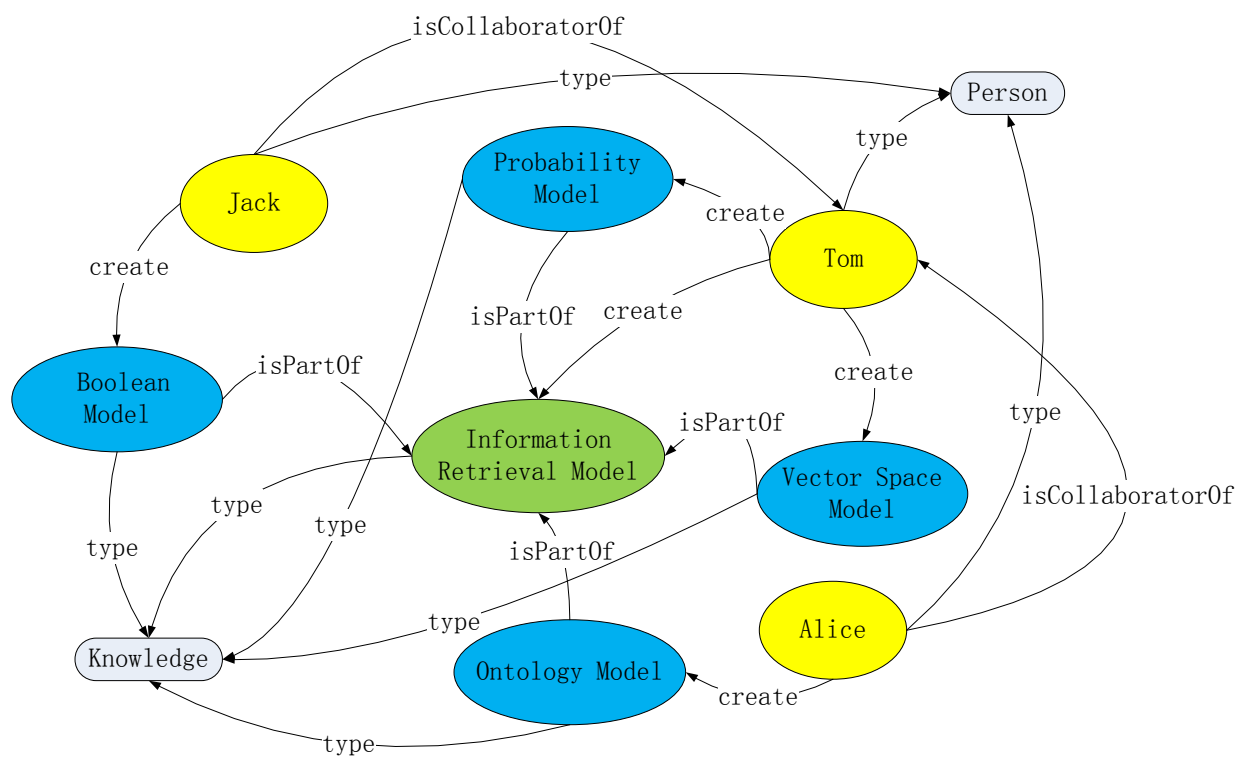

Figure 5. An example of SKN

Figure 6 depicts part of serialization of the example SKN in Resource Description Framework (RDF/XML). In Figure 6, Ontology_Model, Boolean_Model, Probality_Model, Vector_Space_Model and Information_Retrieval_Model are instances of the class Knowledge while Alice, Tom and Jack are instances of the class Person.

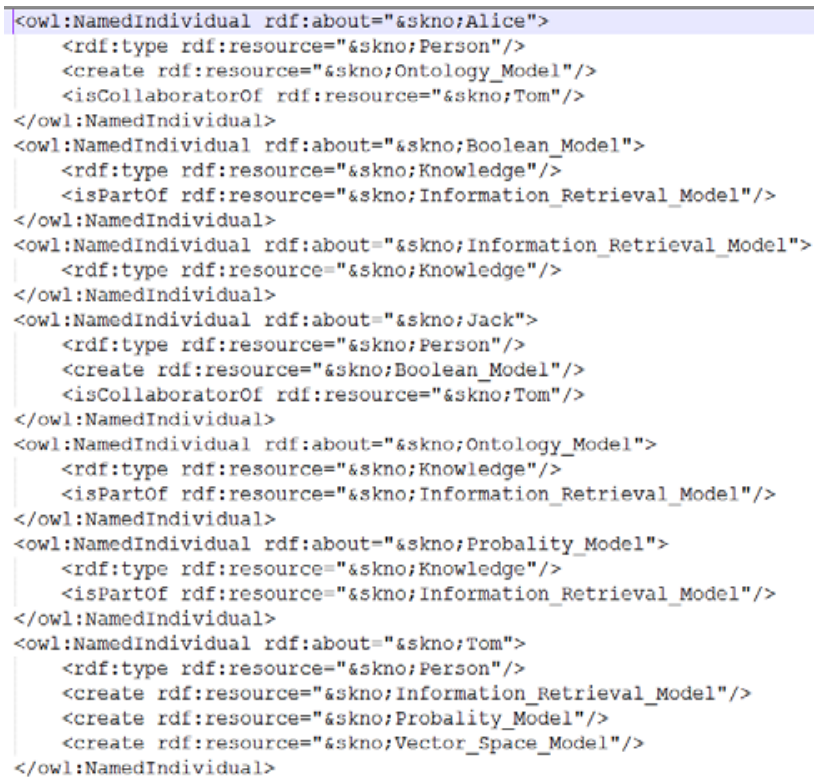

Figure 6. Serialization of the example of SKN
The representation of Right Persons (RPs) is the core function of the RPs Generator. In OKCs, person as an entity has many characteristics from what learning can offer. From the perspective of knowledge, RPs should have the higher degree of correlation in the specific domain knowledge. From the perspective of knowledge quality, RPs should have the higher degree of knowledge authority. Meanwhile, the availability and reputation of the RPs are the important factors for learners. The usability of the RPs is also the important characteristic. These characteristics are key factors that play the core role in the representation of RPs.

Taking into consideration of the above five characteristics of the RPs, we define five parameters (as shown in Table 2), to represent RPs including knowledgerelated degree (KRD), knowledge-authority degree (KAD), social reputation degree (SRD), community availability degree (CAD), and community usability degree (CUD). According to five parameters model of RPs representation, five two-dimensional matrixes (KRD matrix, KAD matrix, SRD matrix, CAD matrix and CUD matrix) are created to represent RPs and relationships between RPs and knowledge.

Table 2. Five parameters definition

\begin{tabular}{lll}
\hline Number & Parameter & Description \\
\hline 1 & KRD & knowledge-related degree \\
2 & KAD & knowledge-authority degree \\
3 & SRD & social reputation degree \\
4 & CAD & community availability degree \\
5 & CUD & community usability degree \\
\hline
\end{tabular}


RPs Generator can generate the five two-dimensional matrixes employing hybrid technologies base on SKN. For the matrix of KRD, we use an expert finding approach base on topic mode (LDA) to compute the relevance between RPs and knowledge. For the matrix of SRD, we use social network analysis techniques (SNA) to compute the social reputation of RPs. For the matrix of KAD, semantic link analysis techniques are used to compute knowledge-authority of RPs. For the CAD matrix and CUD matrix, we use SPARQL to get data from SKN.

\subsection{Query Semantic Parser}

Semantic Parsing is the process of converting query into a formal and logical meaning representation to understand learners' real learning needs. Query Semantic Parser is designed to get semantic information from the learners' request using the general ontologies and domain ontologies that is aimed at enriching search query information.

\subsection{Semantic Matching}

Semantic Matching is aimed at computing semantic relevance between the output of Query Semantic Parser and the characteristics of RPs generated by RPs Generator. The LDA mode is utilized in the process of the semantic similarity measures of Semantic Matching.

\subsection{Semantic Reasoner}

Semantic Web Rule Language (SWRL) is an expressive rule language that enables users to write rules that can be expressed in terms of OWL concepts to provide powerful logical reasoning capabilities [33]. Semantic Reasoner is designed to find new relationships in SKN. A SWRL rule is defined as follows: isCollaboratorOf(?x, ?y) $\wedge$ isCollaboratorOf(?z, ?y)-> $\operatorname{Peer}(? x, ? \mathrm{z})$.

In this rule, isCollaboratorOf(?x, ?y) represents that ?x is collaborator of ?y and $\operatorname{Peer}(? x, ? z)$ represents that $? x$ is peer of ?z. If the relationship between isCollaboratorOf(?x, ?y) and isCollaboratorOf(?z, ?y) is satisfied, Semantic Reasoner can then get new relationship Peer(?x, ?z) . Using the above rule, Semantic Reasoner can get latent semantic relationship in SKN and find new relations among persons to get more social network information in SKN.

\subsection{RPs Ranker}

After the process of Semantic Matching and Semantic Reasoner, the RPs Ranker gets the calculation result to rank the RPs according to the five parameters model of RPs representation, so as to evaluate the RPs from five perspectives. The RPs Ranker can provide different rank lists according to different learners' requirements.

\subsection{RPs Finding GUI}

RPs Finding GUI is a graphic user interface for accepting learners' query and providing multi-visual right personfinding results. RPs Finding GUI provides two types of finding service: Peers Finding and Experts Finding. Learner can choose the finding services. As for learners' query, it provides friendly search textbox to enable learner input query request in the format of keywords or natural languages. It also offers quick search templates and advanced search functions for learners to satisfy learners' different needs. After inputting, RPs Finding GUI provides finding knowledge-relevant persons with two different views: list view and network view.

\section{Learning Application Scenarios}

Here we assume that learner A is a primary school teacher who will teach the Chinese language. He is a novice teacher and is preparing his first course of "Sun Moon Lake". Due to the lack of teaching experience, learner A needs to find the related peers and experts who have the teaching experience of courses similar to "Sun Moon Lake”.

At first, learner A logs in to an online and open knowledge community called the Learning Cell Knowledge Community (http://lcell.bnu.edu.cn/) and searches "Sun Moon Lake" using the functions provided by the traditional full-text search engine. It is disappointing that the system returns more than 200 related results. At this time, facing so many people, learner $\mathrm{A}$ is confused and does not know who would be the right ones.

As a result, learner A begins to use the intelligent people-finding system (IRPFS) base on the RPFinder to search with the keywords "Sun Moon Lake". The IRPFS provides learner A with high quality social network services including people with the right knowledge and experiences. The search results provided by the IRPFS are based on the intelligent calculation of the RPFinder. Learner A can learn and communicate with related experts and peers. At the same time, he can find more related knowledge from them and ask for recommendations of more experts and peers when needed.

\section{Conclusions}

In open knowledge communities, finding right persons is vital for learners to engage in smart learning. If they can easily find the right experts and peers, they can get the channels to gain knowledge and communication opportunities. This paper introduces the design of an intelligent framework for finding right persons in OKCs. 
Considering the social interaction characteristic of OKCs, a novel SKN model is proposed to represent the integration space of social network and knowledge network using the ontologies and linked data technologies. Based on the SKN construction, functions and mechanism of the modules of RPs Generator, Query Semantic Parser, Semantic Matching, Semantic Reasoner, RPs Ranker and RPs Finding GUI are designed and expounded. The learning application scenarios of the intelligent framework will also be demonstrated in our future educational applications.

For future work, we will develop and realize the intelligent right persons finding system in our Learning Cell Knowledge Community to provide intelligent finding service for learners. The IRPFS will be implemented based on many open-source ontology editors and development tools such as Protégé, Jena, SWRLTab, Jena TDB and so on. By Comparing with the traditional search service, we will test and evaluate the RPFinder in different learning context using both qualitative and quantitative methods.

\section{Acknowledgements}

This research is funded by the program "Mobile Learning Platform Development and Application Demonstration”. More information about the project is available at: http://mllab.bnu.edu.cn/. We sincerely thank all reviewers and the Chief editor Minjuan Wang for her thorough editorial work. This paper is therefore also supported by the Oriental Scholar Program of Shanghai Municipal Education Committee (TPKY052WMJ).

\section{References}

[1] YANG XM, QIU Q, Yu SQ, HASAN T. (2014) Designing a trust evaluation model for open-knowledge communities, British Journal of Educational Technology, 45(5), 880-901.

[2] BRUCKMAN A. (2002) The future of e-learning communities, Communications of the ACM, 45 (4), 60-63.

[3] TAURISSON N, TCHOUIKINE P. (2004) Supporting a learner community with software agents, Educational Technology \& Society, 7 (2), 82-91.

[4] AVOURIS N, KOMIC V, MARGARITIS M, FIOTAKIS G. (2004) An environment for studying collaborative learning activities, Educational Technology \& Society, 7 (2), 34-41.

[5] YANG SJH, CHEN IYL, KINSHUK, CHEN NS. (2007) Enhancing the quality of e-learning in virtual learning communities by finding quality learning content and trustworthy collaborators, Educational Technology and Society, 10(2), 84-95.

[6] YANG C, CHANG YS. (2011) Assessing the effects of interactive blogging on student attitudes towards peer interaction, learning motivation, and academic achievements, Journal of Computer Assisted Learning, 28, 126-135.

[7] CHARITONOS K, BLAKE C, SCANLON E, JONES, A. (2012) Museum learning via social and mobile technologies: (How) can online interactions enhance the visitor experience? British Journal of Educational Technology, 43(5), 802-819.
[8] TREC. Enterprise track. (2005) Retrieved from http://www.ins.cwi.nl/projects/trec-ent/wiki/.

[9] YIMAM D. (1996) Expert finding systems for organizations: Domain analysis and the demoir approach, In ECSCW 999 Workshop: Beyond Knowledge Management: Managing Expertise, New York, NY, USA. ACM Press, 276-283.

[10] YIMAM-SEID D, KPNDS A. (2003) Expert finding systems for organizations: Problem and domain analysis and the demoir approach, In Journal of Organizational Computing and Electronic Commerce, 13(1), 1-24.

[11] ZHANG J, ACKERMAN M, ADAMIC L. (2007) Expertise networks in online communities: Structure and algorithms, In Proceedings of the 16th International Conference on World Wide Web, ACM, 221-230.

[12] BALOG K, DE RIJKE M. (2007) Determining expert profiles (with an application to expert finding), In IJCAI'07: Proceedings of the 20th international joint conference on artificial intelligence, 2657-2662.

[13] BALOG K, AZZOPARDI L, DE RIJKE M. (2009) A language modeling framework for expert finding, Information Processing and Management, 45, 1-19.

[14] SERDYUKOV P, HIEMSTRA D. (2008) Modeling documents as mixtures of persons for expert finding, In 30th European conference on information retrieval (ECIR ), 309-320.

[15] ZHOU GY, ZHAO J, HE TT, WU WS. (2014) An empirical study of topic-sensitive probabilistic model for expert finding in question answer communities, Knowledge-Based Systems, 66, 136-145.

[16] JURCZYK P, AGICHTEIN E. (2007) Discovering authorities in question answer communities by using link analysis, In CIKM'07: Proceedings of the 16the international conference on information and knowledge management. Lisboa, Portugal: ACM Press.

[17] DORN J, NZA T, PICHLMAIR M. (2007) Ontology development for human resource management, In Proceedings of 4th International Conference on Knowledge Management, Series on Information and Knowledge Management, 109-120.

[18] ALEMAN-MEZA B, BOJARS U, BOLEY H, BRESLIN J, MOCHOL M, NIXON L, POLLERES A, ZHDANOVA A. (2007) Combining rdf vocabularies for expert finding, In Proceedings of the 4th European Semantic Web Conference (EWSC).

[19] GOMEZ-PEREZ A, RAMIREZ J, VILLZAONTERRAZAS B. (2007) An ontology for modelling human resources management based on standards, In Proceedings of the 11th international conference, KES 2007 and XVII Italian workshop on neural networks conference on Knowledge-based intelligent information and engineering systems: Part I, Springer-Verlag, 534-541.

[20] LIU DR, CHEN YH., KAO WC, WANG HW. (2013) Integrating expert profile, reputation and link analysis for expert finding in question-answering websites, Information Processing and Management ,49, 312-329.

[21] WANG GA, JIAO J, ABRAHAMS AS. FAN WG, ZHANG ZG. (2013) ExpertRank: A topic-aware expert finding algorithm for online knowledge communities, Decision Support Systems, 54, 1442-1451.

[22] LI Y, MA S, ZHANG Y, HHUANG R. (2012) Expertise network discovery via topic and link analysis in online communities, Proceedings of the $12^{\text {th }}$ International Conference on Advanced Learning Technologies , Rome, Italy, 311-315. 
[23] O’REILLY, T. (2005) What is Web 2.0 - Design patterns and business models for the next generation of software. Retrieved from http:/www.oreillynet.com/pub/a/oreilly/tim/news/2005/09/ 30/what-is-web-20.html.

[24] JOVANOVIC J., GASEVIC D., TORNIAI C., BATEMAN, S., HATALA, M. (2009) The social semantic web in intelligent learning environments - State of the art and future challenges. Interactive Learning Environments, 17(4), 273-309.

[25] SCHAFFERT S, HILZENSAUER, W. (2008) On the way towards personal learning environments: Seven crucial aspects. eLearning Papers, 9, 1-10.

[26] MIKROVYANNIDIS, A. (2007) Toward a social semantic web. IEEE Computer, 40(11), 113-115.

[27] HEFLIN J, HENDLER JA. (2001) Portrait of the semantic web in action. IEEE Intelligent Systems, 16 (2), 54-59.

[28] JOVANOVIC J, GASEVIC D, BROOKS C, DEVEDZIC V, HATALA M, EAP T. (2007) Using semantic web technologies to analyze learning content. IEEE Internet Computing, 11(5), 45-53.

[29] BRESLIN J, DECKER S. (2006) Semantic Web 2.0: Creating social semantic information spaces. Tutorial at the World Wide Web conference 2006, Edinburgh, Scotland. Retrieved from http://www2006.org/tutorials/\#T13

[30] GRUBER T. (2008) Collective knowledge systems: Where the social web meets the semantic web. Web Semantics: Science, Services and Agents on the World Wide Web, 6(1), 4-13.

[31] Wasserman, Stanley and Katherine B. Faust. (1994) Social Network Analysis: Methods and Applications. New York: Cambridge University Press.

[32] BERNERS-LEE T. (2006) Linked Data. Retrieved from http://www.w3.org/DesignIssues/LinkedData.html.

[33] SWRL: A Semantic Web Rule Language Combining OWL and RuleML. (2004) Retrieved from http://www.w3.org/Submission/SWRL/. 